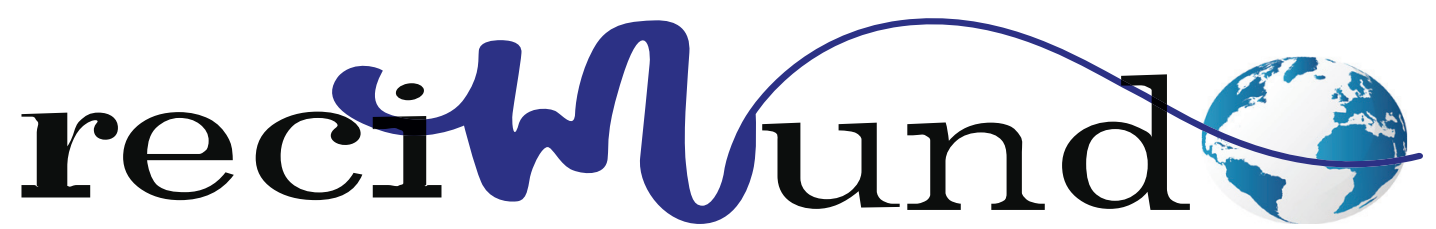

Revista Científica Mundo de la Investigación y el Conocimiento

DOI: 10.26820/recimundo/4.(4).octubre.2020.270-281

URL: http://recimundo.com/index.php/es/article/view/906

EDITORIAL: Saberes del Conocimiento

REVISTA: RECIMUNDO

ISSN: 2588-073X

TIPO DE INVESTIGACIÓN: Artículo de Revisión

CóDIGO UNESCO: Ciencias Médicas

PAGINAS: 270-281

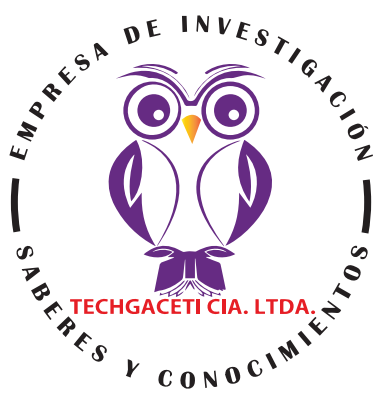

\title{
Síndrome de Parkinson: Revisión bibliográfica y actualización
}

Parkinson's syndrome: bibliographic review and update Síndrome de Parkinson: revisão bibliográfica e atualização

Cristhian Rubén Vallejo Zambrano; Ricardo Aspren Jiménez Jiménez²; Vanessa Estefania Morán Rodríguez;; Marcela Elizabeth Gómez Chumo4; María Belén Del Valle Pilay5; Néstor Jamil Palma Moreno ${ }^{6}$

\section{RECIBIDO: 10/07/2020 ACEPTADO: 26/08/2020 PUBLICADO: 15/10/2020}

1. Departamento de Investigación y falla cardiaca. INCAP - CICCMA 3er nivel de atención; Estudios para posgrado (EEUU); Médico Cirujano; Manta, Ecuador; mdcardiologycrvz@hotmail.com; iD https://orcid.org/0000-0001-5513-8507

2. Médico Especialista en Neurocirugía IESS Manta - Manabí / Manta Hospital Center; Neurocirujano - Oncólogo; Miembro de la Sociedad Ecuatoriana de Neurocirugía; Manta, Ecuador; ricardojimenezjimenez@hotmail.com; iD https://orcid.org/00000001-9910-6523

3. Departamento de Investigación y falla cardiaca; Médico Cirujano; Manta, Ecuador; vaesmoro@outlook.com; (iD https:// orcid.org/0000-0001-6785-4404

4. Estudios para posgrado (EEUU); Médico Cirujano; Queens, New York, Estados Unidos; megomez@udlanet.ec; (DD https:// orcid.org/0000-0002-2876-1819

5. Médico General Asistencial Hospital Rodríguez Zambrano; Asistente en Neurocirugía Manta Hospital Center; Médico Cirujano; Manta, Manabí, Ecuador; beldelvalle@live.com; (D) https://orcid.org/0000-0001-8214-1111

6. Asistente en Neurocirugía Manta Hospital Center; Médico Cirujano; Manta, Manabí, Ecuador; drnestorpalma@hotmail.com; (iD) https://orcid.org/0000-0002-7480-2554

CORRESPONDENCIA

Cristhian Rubén Vallejo Zambrano

mdcardiologycrvz@hotmail.com

Manta, Ecuador

๑) RECIMUNDO; Editorial Saberes del Conocimiento, 2020 


\section{RESUMEN}

El síndrome de Parkinson (SP), es un desorden neurodegenerativo causado por una pérdida progresiva de neuronas dopaminergicas en la Sustancia Nigra pars compacta (SNpc). SP ocasiona ciertas anomalías motoras como por ejemplo temblor en reposo y rigidez muscular. Con un buen diagnóstico y en base a su fisiopatología podemos conseguir mejores resultados dando un tratamiento tanto clínico como quirúrgicos según corresponda. En la actualidad, debido a nuevas formas de tratamiento para el SP, debemos conocer las bases fisiopatológicas del mismo, para posterior analizar de una manera más estricta la terapéutica a seguir. El objetivo de esta revisión es integrar y plasmar las teorías fundamentales y actuales relacionadas al Síndrome de Parkinson. El estudio se realizó mediante un búsqueda minuciosa y análisis de artículos indexados en las bases de información médicas: Pubmed, Pubmed Central, Medline, por lo que es un estudio tipo documental, bibliográfico. Es fundamental tener un estudio bibliográfico, en el cual se pueda comprender claramente la fisiopatología y así, poder diferenciar las nuevas tendencias en cuanto al tratamiento clínico o quirúrgico que puedan tener los pacientes con (SP).

Palabras clave: Parkinson; Fisiopatología; Diagnostico; Tratamientos.

\section{ABSTRACT}

Parkinson's syndrome (PS) is a neurodegenerative disorder caused by a progressive loss of dopaminergic neurons in the substantia nigra pars compacta (SNpc). PS cause certain motor abnormalities such as resting tremor and muscle stiffness. With a good diagnosis and based on its pathophysiology we can achieve better results by giving both clinical and surgical treatment as appropriate. At present, due to new forms of treatment for PS, we must know the pathophysiological bases of it, to later analyze in a stricter way the therapy to be followed. The objective of this review is to integrate and translate the fundamental and current theories related to Parkinson's Syndrome. The study was carried out through a meticulous search and analysis of articles indexed in the medical information databases: Pubmed, Pubmed Central, Medline, so it is a documentary, bibliographic type study. It is essential to have a bibliographic study, in which the pathophysiology can be clearly understood and thus, to be able to differentiate new trends in terms of clinical or surgical treatment that patients with (PS) may have.

Keywords: Parkinson; Pathophysiology; Diagnosis; Treatments.

\section{RESUMO}

A síndrome de Parkinson (PS) é uma doença neurodegenerativa causada por uma perda progressiva de neurônios dopaminérgicos na pars compacta da substância negra (SNpc). PS causa certas anormalidades motoras, como tremor de repouso e rigidez muscular. Com um bom diagnóstico e com base na sua fisiopatologia, podemos alcançar melhores resultados, administrando o tratamento clínico e cirúrgico conforme apropriado. Atualmente, devido às novas formas de tratamento da SP, devemos conhecer as bases fisiopatológicas da mesma, para posteriormente analisarmos de forma mais rigorosa a terapêutica a ser seguida. O objetivo desta revisão é integrar e traduzir as teorias fundamentais e atuais relacionadas à Síndrome de Parkinson. O estudo foi realizado por meio de busca e análise criteriosa de artigos indexados nas bases de dados de informações médicas: Pubmed, Pubmed Central, Medline, portanto, é um estudo do tipo documental, bibliográfico. É imprescindível um estudo bibliográfico, no qual a fisiopatologia possa ser bem compreendida e, assim, poder diferenciar as novas tendências em termos de tratamento clínico ou cirúrgico que os pacientes com (SP) podem ter.

Palavras-chave: Parkinson; Fisiopatologia; Diagnóstico; Tratamentos. 


\section{VALLEJO ZAMBRANO, C. R., JIMÉNEZ JIMÉNEZ, R. A., MORÁN RODRÍGUEZ, V. E., GÓMEZ CHUMO, M. E., DEL VALLE PILAY, M. B., \& PALMA MORENO, N. J.}

\section{Introducción}

\section{Síndrome de Parkinson}

Es una enfermedad crónica degenerativa que se caracteriza por una muerte temprana de las neuronas dopaminérgicas en la Sustancia Nigra pars compacta con presencia de alfa sinucleina que es una proteína intracelular [1].

En esta revisión bibliográfica discutiremos la epidemiología, características clínicas, patofisiología, diagnóstico, manejo (clínico o quirúrgico).

\section{Epidemiología}

El síndrome de Parkinson considerado como el segundo trastorno neurodegenerativo más común que constituye la principal causa de discapacidad neurológica [2]. La prevalencia del Parkinson se ha duplicado en los últimos 26 años, según (the lancet neurology), este estudio incluyó a varias zonas geográficas a nivel mundial donde detallaba un aumento de 2.1 millones en 1960 a 6.1 millones de casos para el 2016 [3], sugieren investigadores que el envejecimiento es un dato de relevancia ya conocido para el aumento de dicha incidencia pero que existen otras posibles causas que podrían describir mejor dicho suceso así como factores de carácter ambiental relacionados con el crecimiento industrial [4] de la misma manera la asociación del abandono del tabaco que se relaciona con aumento de nuevos casos, según estudios.[5]

En la actualidad no se ha obtenido ningún dato sobre incidencia o prevalencia publicado en nuestro país, pero sin embargo en un estudio realizado con 116.983 habitantes de la provincia de Manabí, 317 fueron considerados como probables casos para el desarrollo del síndrome de Parkinson; pero únicamente 285 (0,24\%) fueron diagnosticadas de SP, basándose en criterios del Banco de Cerebro de Londres. Con respecto al sexo, no se demostró diferencia considerable; sin embargo, se observó mayor prevalencia por el sexo masculino $(56,14 \%)$ en relación con el sexo femenino $(43,86 \%)$. [6]

\section{Fisiopatología}

El síndrome de Parkinson tiene como sustrato neuropatológico la perdida de forma selectiva de neuronas dopaminergicas localizadas en la pars compacta de la sustancia nigra (SNpc) [7] a causa de la degradación proteosomal inadecuada de la proteína alfa-sinucleína, lo que condiciona su depósito en el citoplasma de las neuronas, como inclusiones llamadas los cuerpos de Lewy. [8]

Dichos depósitos presentan una división en 6 estadios, inicialmente presentan afectación neuronal de la medula oblongada, el bulbo olfatorio y el núcleo dorsal del vago, posteriormente se comprometen en el proceso al núcleo cerúleo, caudal del rafe y a las neuronas reticulares que conforman el núcleo gigantocelular. El tercer estadio se involucran las neuronas de la Sustancia Nigra pars compacta en los últimos tres estadios se provoca una afectación que ocurre en forma en secuencial de las estructuras de la corteza límbica, las áreas corticales de asociación, especialmente la prefrontal y por último las denominadas cortezas motoras y sensoriales primarias. [9][10]

La ausencia de las neuronas dopaminérgicas va a condicionar un desbalance en la neurotransmisión estriatal además de otras estructuras localizados en los ganglios básales, los mismo que son responsable de una alteración de la actividad neuronal en los principales núcleos de salida de los ganglios basales (Globo Pálido interno (GPi) y sustancia nigra pars reticulata (SNpr).

A consecuencia de dichas alteraciones se sobreinhibe el tálamo motor y por esta vía se oponen a la facilitación del movimiento corticalmente generado, lo que constituye 
la base de la sintomatología de la enfermedad. [11]

Albin, Young y Penny en 1989 propusieron la división del circuito motor en dos principales sistemas de proyección en base a poblaciones neuronales diferentes en el putamen que llegan hasta los núcleos de salida por medio de rutas diferentes, quedando segregado en un circuito directo y uno indirecto. [12][13]

El circuito motor directo es monosináptico y presenta un génesis neuronal estriatales gabaérgicas que expresan sustancia $P$, dinorfina y receptores dopaminérgicos de la familia D1, cuyas elongaciones realizan sinapsis directa en los núcleos de salida (GPi y SNpr). [14]

A diferencia, el circuito motor indirecto tiene un comienzo neuroal gabaérgicas putaminales que otorgan en-cefalina y que tiene receptores dopaminérgicos D2, expuesto en sus axones al globo pálido externo (GPe). [12] Este núcleo a su vez envia los axones al núcleo subtalámico (NST) expresando (GABA) como neurotransmisor. Las neuronas de proyección del NST que expresan ácido glutámico como neurotransmisor van a realizar sinapsis con el GPi y la SNpr. [12] [13] Proyecciones de los núcleos de salida son gabaérgicas y provocan relevo sináptico en los núcleos ventral anterior y ventro - lateral del tálamo antes de seguir su ruta a las áreas corticales que le dan origen, finalizando así el circuito córtico-ganglios basales-tálamo-cortical.

Se ha demostrado que el parkinsonismo tiene relación con hiperactividad del NST provocando hiper sincronización de las oscilaciones de baja frecuencia entre el NST y el Globo Pálido (GP), lo que condiciona con una interferencia con la capacidad de iniciar o detener programas motores en la corteza. [15][16]
Por un lado, los NST expresan como neurotransmisor al AG considerado como agente altamente neurotóxico en algunas circunstancias, en particular cuando existe una falla en el metabolismo oxidativo, así como en la enfermedad de Parkinson. Las principales referencias del NST van direccionadas a los núcleos de salida de los GB pero a al mismo tiempo proyecta a la SNpc, esta última proyección no es tan densa, pero cobra especial interés en el contexto de la neuroprotección en el síndrome de Parkinson, por lo que las neuronas dopaminérgicas supervivientes en la SNpc de los pacientes con síndrome de Parkinson y estarían representadas en concentraciones elevadas de AG resultante por la hiperactividad neuronal del NST. [17][18]

La consecuencia de esta línea de analitica sería que la terapéuticas encaminada a frenar la hiperactividad del NST, además de tener una acción antiparkinsoniana sintomática, las que podrían tener un efecto neuroprotector indirecto. [18]

\section{Manifestaciones clínicas}

El síndrome de Parkinson se define por la presencia de bradiquinesia junto con al menos una de las siguientes manifestaciones: rigidez muscular, temblor en reposo o inestabilidad postural. [20]

Para la clasificación clínica se subdivide en síntomas motores y síntomas no motores los cuales se encuentran con mayor prevalencia en estos pacientes, y pueden iniciar antes que los síntomas motores. [21]

Entre los síntomas no motores podemos encontrar: sueño ligero, trastorno del comportamiento del sueño con movimientos oculares rápidos, somnolencia en horas del día, hiposmia y disturbios en la función autonómica (hipotensión orto estática, disfunción urogenital, constipación), deterioro cognitivo, trastornos del estado de ánimo y dolor. [21]

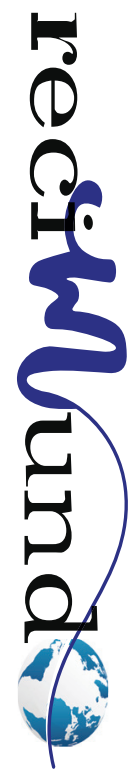


VALLEJO ZAMBRANO, C. R., JIMÉNEZ JIMÉNEZ, R. A., MORÁN RODRÍGUEZ, V. E., GÓMEZ CHUMO, M. E., DEL VALLE PILAY, M. B., \& PALMA MORENO, N. J.

Observaciones clínicas sugieren la presencia de 2 subtipos principales: SP temblor dominante los cuales tienen una progresión lenta, comparada con SP con temblor no dominante (síndrome acinético-rígido) el cual es de progresión más rápida. [22] Según un estudio casi el $90 \%$ de los pacientes con SP con síntomas no motores no responden a la terapia con dopamina. [23]

En cuanto a los síntomas autonómicos estos incrementan con la edad. En relación entre la demencia y el Parkinson es muy estrecha y además se sabe que del $60 \%$ de pacientes con SP desarrollan demencia en aproximadamente 12 años de ser diagnosticados de SP. [24]

\section{Diagnóstico}

El diagnóstico temprano del síndrome de Parkinson es un desafío debido a la gran variedad de diagnósticos diferenciales en los que estas enfermedades no están asociadas con la degeneración o déficit de dopamina. [1]

Los criterios clínicos más utilizados son el banco de Cerebros de Reino Unido el cual tiene una precisión elevada de diagnóstico de un $75-95 \%$, el cual se fundamenta en la Tabla 1 : [25][40]

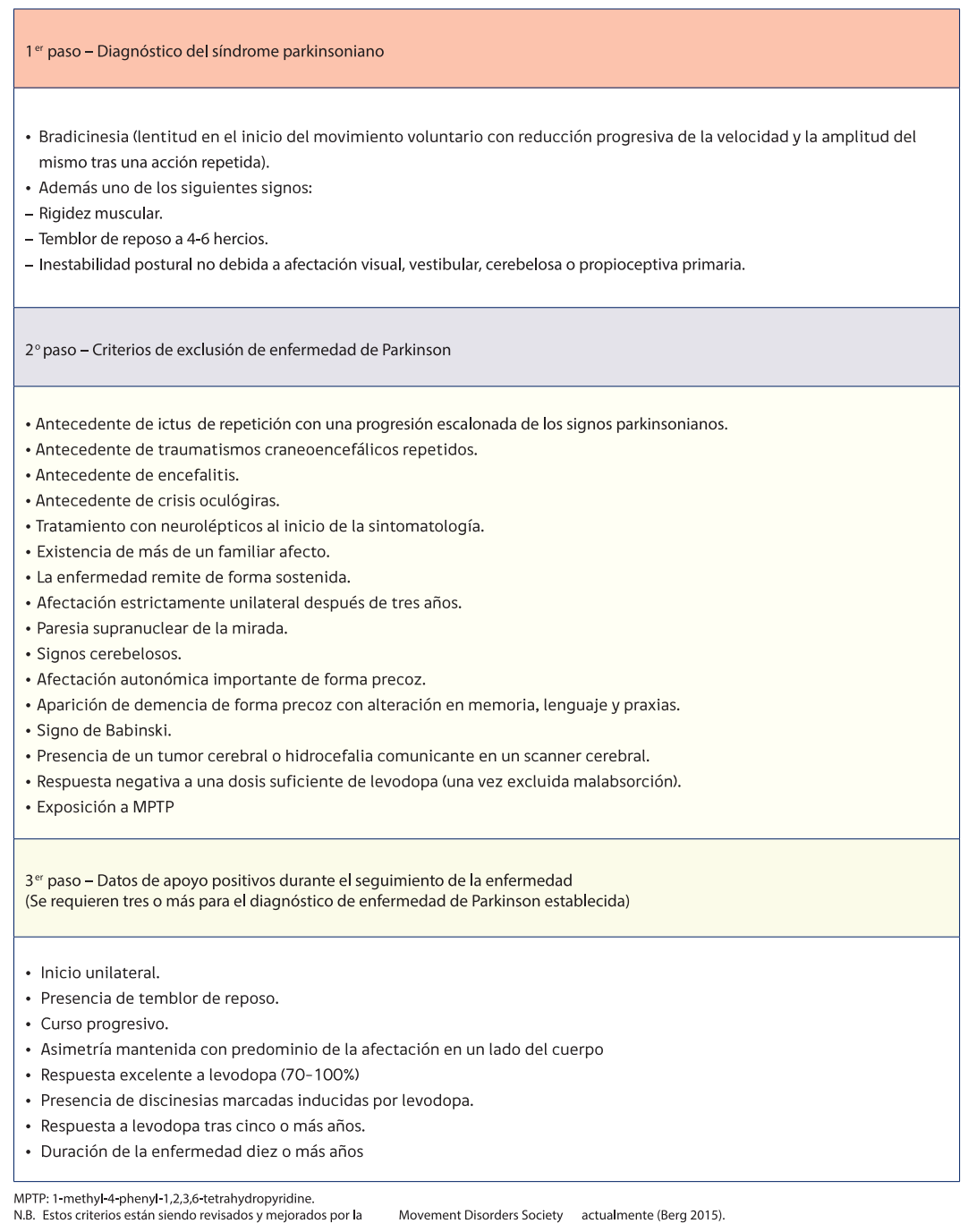

Tabla 1. Banco de Reino Unido para diagnóstico de Parkinson.

Fuente: [25][40] 
Los criterios clínicos son muy importantes ya que con ellos se consigue un $80 \%$ del diagnóstico de este síndrome [26], pero de igual manera los estudios de imagen son vitales para poder confirmar la enfermedad. Entre los estudios de imagen de más relevantes están:

- Tomografía computarizada por emisión de fotón único con 123l-ioflupano: Evalúa la densidad de los terminales dopaminergicos pre sináptico. [27]

- Tomografía por emisión de positrones (PET) con 18 F-DOPAL-6-fluoro-3, 4-dihidroxifenilalnina (18 F-DOPA) la cual evalúa la integridad dopaminergica presináptica. [1] Se realizo un analisis retrospective en un numero de 27 pacientes quienes se sometieron a Scan Pet 18 F-DOPA, los cuales mostraron una sensibilidad $95.4 \%$, especificidad $100 \%$, valor predictivo positivo $100 \%$, y valor predictivo negativo de $87.5 \%$ [28]

- Imagen por resonancia magnética (RMN) se puede combinar con otras técnicas de imagen para determinar un síndrome de Parkinson en estadío temprano.

En cuando al estudio de líquido cefalorraquídeo no es de mucha utilidad para el diagnóstico de SP. [29]

\section{Tratamiento y terapéutica actual}

Los tratamientos en la actualidad se encuentran enfocados a la parte sintomatológica. [30] Ninguna terapéutica ha mostrado un efecto curativo en los estudios realizados hasta estos momentos. [31] Podemos dividir en 2 secciones el tratamiento farmacológico y tratamiento quirúrgico.

\section{Tratamiento farmacológico}

El tratamiento farmacológico podemos subclasificarlo en 2 secciones, las cuales son terapia dopaminergica y terapia no dopaminergica.

\section{Terapia Dopaminergica}

La academia americana de neurología sugiere empezar con el tratamiento una vez que el paciente desarrolle afectación funcional. [32]

Entre las terapias medicamentosas conocidas para el tratamiento de SP con síntomas motores podemos nombrar: levodopa/carbidopa [33], agonistas dopaminergicos los cuales al igual que la levodopa ayuda a disminuir la progresión de la enfermedad [34], inhibidores de la monoamino oxidasa-B, agonistas dopaminergicos inyectables, inhibidores de la catechol-o-metiltransferasa (COMT), inhibidores de los receptores de N-Metil-D-aspartate (NMDA) y anticolinérgicos los cuales ayudan a reducir el temblor [1] [35].

\section{Terapia no dopaminergica (medicación de soporte)}

Se recomienda dar esta terapia a los pacientes con estadio avanzado de SP porque estos tienen poca respuesta a la terapia dopaminérgicas. Esto puede ser debido por anormalidades en otros neurotransmisores como el glutamato, norepinefrina, acetilcolina, o serotonina. [36]

Entre los fármacos de soporte tenemos: Inhibidores de la colinesterasa (demencia) [37], Antidepresivos tricíclicos, inhibidores de la recaptación de serotonina, neurolépticos atípicos, antagonista de los receptores de (NMDA) usado para las disquinesias causadas por la levodopa. [38]

Investigaciones también han demostrado que los inhibidores de c-Abl (Abelson non-receptor tyrosine kinase) pueden prevenir la perdida de neuronas dopaminérgicas y así mejorando el comportamiento motor. [39]

En el siguiente gráfico se encuentran los fármacos usados para el SP. Tabla 2 [40] 
VALLEJO ZAMBRANO, C. R., JIMÉNEZ JIMÉNEZ, R. A., MORÁN RODRÍGUEZ, V. E., GÓMEZ CHUMO, M. E., DEL VALLE PILAY, M. B., \& PALMA MORENO, N. J.

Tabla 2. Fármacos más usados en el manejo del Síndrome de Parkinson

\begin{tabular}{|c|c|c|c|c|}
\hline INDICACIÓN & $\begin{array}{l}\text { DOSIS INICIAL } \\
(\mathrm{MG})\end{array}$ & $\begin{array}{l}\text { POSOLOGÍA } \\
\text { HABITUAL }\end{array}$ & $\begin{array}{c}\text { DOSIS DE } \\
\text { MANTENIMIENTO } \\
\text { (MG/DÍA) }\end{array}$ & $\begin{array}{l}\text { EFECTOS ADVERSOS } \\
\text { MÁS RELEVANTES }\end{array}$ \\
\hline \multicolumn{5}{|c|}{ Manifestaciones motoras } \\
\hline Rasagilina* & 1 & cada $24 \mathrm{~h}$ & 1 & cefaleas, náuseas, pesadillas, $\mathrm{TCl}$ \\
\hline $\begin{array}{l}\text { Levodopa/ } \\
\text { Carbidopa* }\end{array}$ & $50 / 12.5$ & cada $8 \mathrm{~h}$ & $300 / 75-1000 / 100$ & $\begin{array}{l}\text { náuseas, hipotensión, } \\
\text { fluctuaciones motoras }\end{array}$ \\
\hline Rotigotina* & 2 & cada $24 \mathrm{~h}$ & $6-8$ & $\begin{array}{l}\text { náuseas, edema EE.II, } \\
\text { somnolencia, } \mathrm{TCl} \text {, rash }\end{array}$ \\
\hline Ropinirol* & $0,25 \mathrm{SR} \mid 2 \mathrm{ER}, 0$ & $\begin{array}{c}\text { cada } 8 \mathrm{~h} \text { SR | cada } 24 \mathrm{~h} \\
\text { ER }\end{array}$ & $1,5-3 \mid 8-24$ & $\begin{array}{l}\text { náuseas, edema EE.II, } \\
\text { omnolencia, TCI }\end{array}$ \\
\hline Pramipexol* & $0,088 \mathrm{SR} \mid 0,26 \mathrm{ER}$ & $\begin{array}{c}\text { cada } 8 \mathrm{~h} \text { SR } \mid \text { cada } 24 \mathrm{~h} \\
\text { ER }\end{array}$ & $1,1-3,3 \mid 1,05-3,15$ & $\begin{array}{l}\text { náuseas, edema EE.Il, } \\
\text { somnolencia, TCI }\end{array}$ \\
\hline $\begin{array}{l}\text { Apomorfina } \\
\text { subcutánea* }\end{array}$ & $1-10$ & según prescripción & $3-30$ & háuseas, hipotensión, $\mathrm{TCl}$, nódulos \\
\hline Entacapona* & 200 & cada $8 \mathrm{~h}$ & $600-2000$ & clínica gastro-intestinal, orina rojiza \\
\hline Tolcapona* & 100 & cada $8 \mathrm{~h}$ & $300-600$ & $\begin{array}{l}\text { hepatotoxicidad (vigilar), } \\
\text { gastrointestinales }\end{array}$ \\
\hline Trihexifenidilo*ף & 1 & cada $8 \mathrm{~h}$ & $5-15$ & $\begin{array}{l}\text { deterioro cognitivo, } \\
\text { gastrointestinales, }\end{array}$ \\
\hline \multicolumn{5}{|c|}{ Disquinesias/Fluctuaciones } \\
\hline Amantadina* & 100 & cada $24 \mathrm{~h}$ & 200 (2 tomas) & $\begin{array}{l}\text { edema, livedo reticularis, insomnio, } \\
\mathrm{TCI}\end{array}$ \\
\hline Safinamida & 50 & cada $24 \mathrm{~h}$ & $50-100$ & náusea, mareo, fatiga, cefalea \\
\hline \multicolumn{5}{|c|}{ Depresión/Ansiedad } \\
\hline Nortriptilina & $20-40$ & cada $8 \mathrm{~h}$ & $30-150$ & $\begin{array}{l}\text { sequedad de boca, estreñimiento, } \\
\text { hipotensión }\end{array}$ \\
\hline Venlafaxina & 75 & cada $12 \mathrm{~h}$ & $150-375$ & $\begin{array}{l}\text { astenia, gastrointestinales, } \\
\text { disfunción sexual }\end{array}$ \\
\hline Paroxetina & 20 & cada $24 \mathrm{~h}$ & 60 & $\begin{array}{l}\text { hipotensión, gastrointestinales, } \\
\text { disfunción sexual }\end{array}$ \\
\hline \multicolumn{5}{|l|}{ Psicosis/TCl ${ }^{1}$} \\
\hline Clozapina & 12.5 & cada $12 \mathrm{~h}$ & $200-300$ & agranulocitosis (vigilar), somnolencia \\
\hline Quetiapina & 25 & cada $12 / 24 h$ & 150 & $\begin{array}{l}\text { somnolencia, hipotensión, } \\
\text { gastrointestinales }\end{array}$ \\
\hline \multicolumn{5}{|l|}{$\mathrm{RBD}^{2}$} \\
\hline Clonazepam & $0,25-0,5$ & por la noche & $0,5-4$ & somnolencia, fatiga, mareo \\
\hline Melatonina & 3 & por la noche & $3-12$ & somnolencia, fatiga, mareo \\
\hline
\end{tabular}




\begin{tabular}{|c|c|c|c|c|}
\hline INDICACIÓN & $\begin{array}{l}\text { DOSIS INICIAL } \\
\text { (MG) }\end{array}$ & $\begin{array}{l}\text { POSOLOGÍA } \\
\text { HABITUAL }\end{array}$ & $\begin{array}{c}\text { DOSIS DE } \\
\text { MANTENIMIENTO } \\
\text { (MG/DÍA) }\end{array}$ & $\begin{array}{l}\text { EFECTOS ADVERSOS } \\
\text { MÁS RELEVANTES }\end{array}$ \\
\hline \multicolumn{5}{|c|}{ Hipotensión ortostática } \\
\hline Fludrocortisona & 0,1 & cada $24 \mathrm{~h}$ & $0,1-0,2$ & $\begin{array}{l}\text { hipokalemia, edema, insuficiencia } \\
\text { cardiaca, hipertensión supina }\end{array}$ \\
\hline Midodrina & 2,5 & cada $8 \mathrm{~h}$ & $7,5-30$ & $\begin{array}{l}\text { parestesias, prurito, hipertensión } \\
\text { supina }\end{array}$ \\
\hline Piridogstismina & 30 & cada $8 \mathrm{~h}$ & $90-180$ & dolor abdominal, náuseas, sialorrea \\
\hline \multicolumn{5}{|c|}{ Disfunción genitourinaria } \\
\hline $\begin{array}{l}\text { Sildenafilo } \\
\text { (disfunción } \\
\text { eréctil) }\end{array}$ & 50 & $\begin{array}{l}30^{\prime}-60^{\prime} \text { antes de } \\
\text { relación }\end{array}$ & 100 & $\begin{array}{l}\text { cefalea, rubefacción, percepción } \\
\text { anormal de colores }\end{array}$ \\
\hline $\begin{array}{l}\text { Trospio (vejiga } \\
\text { hiperactiva) }\end{array}$ & 20 & cada $12 \mathrm{~h}$ & 40 & $\begin{array}{l}\text { sequedad de boca, glaucoma, } \\
\text { taquicardia }\end{array}$ \\
\hline $\begin{array}{l}\text { Darifenacina } \\
\text { (vejiga } \\
\text { hiperactiva) }\end{array}$ & 7,5 & cada $24 \mathrm{~h}$ & 15 & $\begin{array}{l}\text { sequedad de boca, estreñimiento, } \\
\text { visión borrosa }\end{array}$ \\
\hline $\begin{array}{l}\text { Mirabegron } \\
\text { (vejiga } \\
\text { hiperactiva) }\end{array}$ & 25 & cada $24 \mathrm{~h}$ & 50 & $\begin{array}{l}\text { hipertensión arterial, taquicardia y } \\
\text { cefalea }\end{array}$ \\
\hline \multicolumn{5}{|c|}{ Deterioro cognitivo } \\
\hline Rivastigmina & $\begin{array}{l}1,5 \text { (oral) } / 4,6 \\
\text { (parche) }\end{array}$ & cada $12 / 24 \mathrm{~h}$ & $3-12 / 4,6-13,3$ & $\begin{array}{l}\text { náuseas, vómitos, bradicardia, } \\
\text { cefalea }\end{array}$ \\
\hline \multicolumn{5}{|l|}{ Estreñimiento } \\
\hline $\begin{array}{l}\text { Macrogol/ } \\
\text { Polietilenglicol }\end{array}$ & $4 g r$ & por la mañana & $8 g r$ & diarrea, dolor y distensión abdominal \\
\hline Lactulosa & $10-20$ & día & 60 & diarrea, dolor y distensión abdominal \\
\hline
\end{tabular}

1TCl: Trastorno de control de impulsos. Se suele recomendar reducir o eliminar lentamente agonistas dopaminérgicos primero si el paciente toma, antes de empezar con los fármacos descritos 2RBD: Trastorno de conducta de sueño en la fase REM; SR: liberación estándar ( standard release ); ER: liberación retardada ( extended reléase ); EE.II: Extremidades inferiores *Pueden inducir alucinaciones y/o psicosis ๆ Usado para el tratamiento del temblor principalmente.

\section{Fuente: [40]}

\section{Tratamiento Quirúrgico}

Desde hace aproximadamente 70 años (1940) se han realizado estudios acerca del manejo quirúrgico del SP. [41] En los últimos 25 años la estimulación cerebral profunda (ECP) se ha transformado en la terapia quirúrgica predominante. Las indicaciones para las que se usa esta (ECP) son para fluctuaciones motoras, disquinesias, temblor y síntomas por el efecto adverso de la levodopa. [41]
En la actualidad existen nuevos avances en la neuroestimulación para el SP. Desde los inicios con la palidotomía, [42] los objetivos para el tratamiento de síntomas motores han sido los ganglios de la base. Entre los objetivos principales en estas cirugías tenemos el segmento interno del pallidum (GPI), y el núcleo subtalámico, e incluso hasta el tálamo en algunos casos. [43]

Estimulación cerebral profunda no invasiva. Debido a que la (ECP) necesita de una cirugía abierta, se han ido descubriendo nue- 
VALLEJO ZAMBRANO, C. R., JIMÉNEZ JIMÉNEZ, R. A., MORÁN RODRÍGUEZ, V. E., GÓMEZ CHUMO, M. E., DEL VALLE PILAY, M. B., \& PALMA MORENO, N. J.

vas técnicas menos invasivas para aplicar la neuroestimulación como es el caso del procedimiento en el que se usa un ultrasonido enfocado, [44] el cuál ha sido aprobado para el temblor esencial, pero últimamente se lo está usando en el tratamiento del SP con subtalamotomías y palidotomías. [45]. Por otro lado, tenemos la estimulación magnética transcraneal (EMT) que es otro método de estimulación cerebral no invasiva, pero este es incapaz de modular los objetivos cerebrales profundos como los ganglios basales. [46]

Se ha realizado un algoritmo para la secuencia del tratamiento del síndrome de Parkinson [46], el mismo que se presentará a continuación:

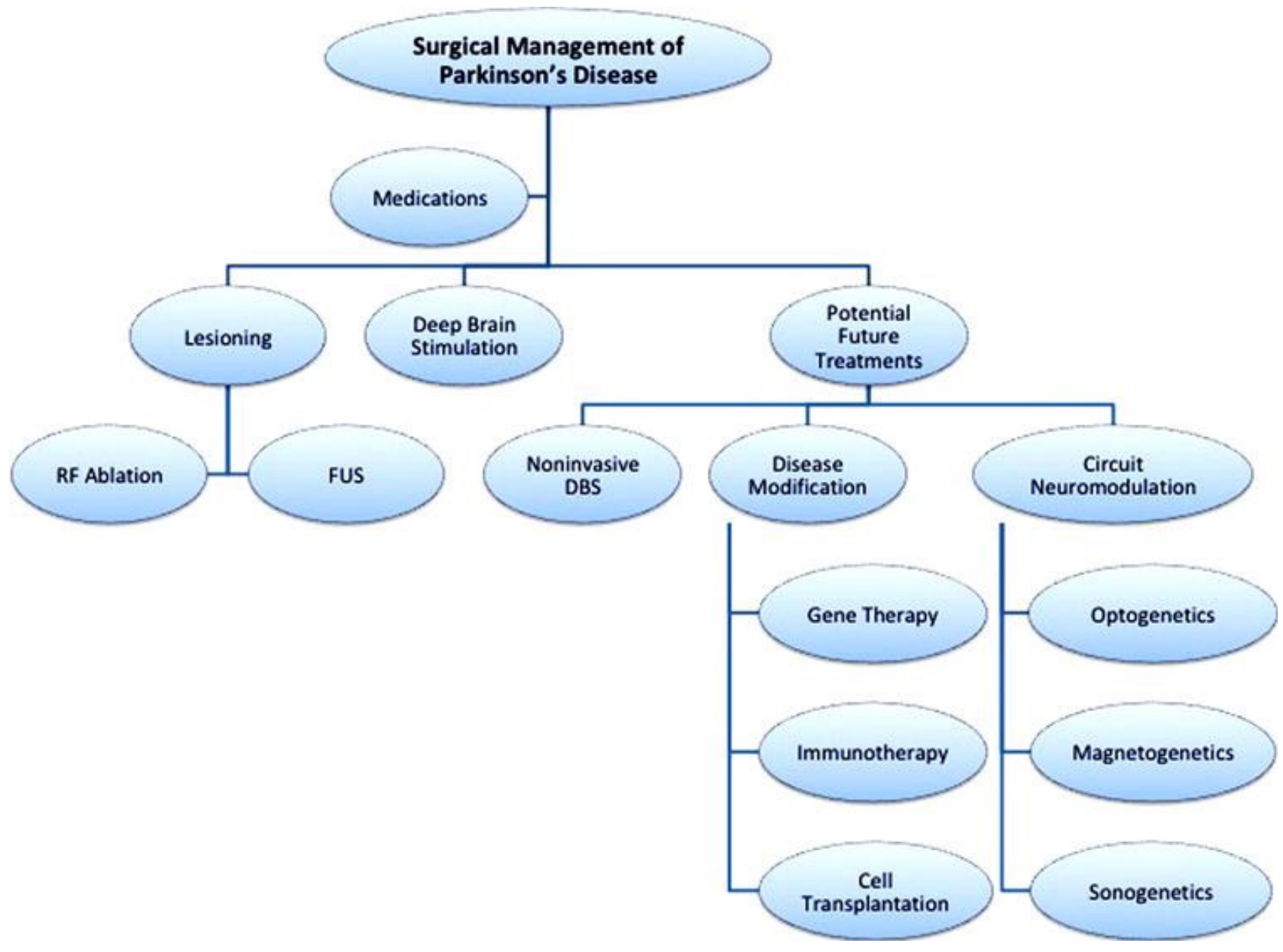

Algoritmo 1. Nuevos tratamientos actuales y potenciales de la enfermedad de Parkinson.

Fuente: [46] 


\section{Conclusión}

El Síndrome de Parkinson (SP) es una de las enfermedades neurodegenerativas más frecuentes que afecta a la humanidad, aumentando así la morbilidad y mortalidad, transformándose en un grave problema de la salud comunitaria.

Conocer sobre ella, sus manifestaciones clínicas, la forma adecuada para conseguir el diagnóstico, y conocer el tratamiento a seguir es de vital importancia, debido a que como médicos de salud pública y especialistas debemos otorgar a nuestros pacientes una mejor atención, con tratamiento que se adapte a las condiciones económicas de ellos, y con eso prolongar la vida con una mejor calidad, disminuyendo la progresión de esta enfermedad, ya que al no tener una cura definitiva, mejorar la calidad de vida en nuestros pacientes es el objetivo principal.

\section{Bibliografía}

1. Radhakrishnan DM, Goyal V. Parkinson's disease: A review.Neurol India 2018;66:26-35

2. Adams RD, Victor MD, Allan MD. In: ED McGraw-HiII interamericana, editor. Principios de Neurología. Sexta edición ed. México: 2000. p. 925-31.

3. GBD 2016 Parkinson Disease Collaborators. Global, regional, and national burden of Parkinson's disease, 1990-2016: a systematic analysis for the Global Burden of Disease Study 2016. Lancet Neurol. 2018; 17: 939-53

4. Palacios N. Air pollution and Parkinson's disease-evidence and future directions. Reviews on environmental health. Rev Environ Health 2017; 32 (4): 303-13

5. Costa P, Zabetian C, Ching S, Agarwa P, Yearout D, Checkoway H. Smoking and haptoglobin phenotype modulate serum ferritin and haptoglobin levels in Parkinson disease. J Neural Transm 2016; 123: 1319-30.

6. Juan M, Paola M, Lisbeth A. Elba I, Diana M, (2017). Prevalencia de la Enfermedad de Parkinson: Estudio Puerta-Puerta en la Provincia de Manabí-Ecuador. Prevalence of Parkinson's Disease: Door-to-door Study in Manabi-Ecuador. Revista ecuatoriana de neurologia; 1-3
7. Federico E. Enfermedad de Parkinson y trastornos relacionados. Argentina: 1998.

8. Lowe J, Lennox G, Leigh PN. In: Greenfield's Neuropathology, editor. Disorders of mevement and system degerations. sexta edición ed. Londres: 1996. p. 281-366.

9. Braak H, Del TK, Rub U, de Vos RA, Jansen Steur EN, Braak E. Staging of brain pathology related to sporadic Parkinson's disease. Neurobiol Aging 2003; 24 (2): 197-211.

10. Braak H, Ghebremedhin E, Rub U, Bratzke $H$, Del TK. Stages in the development of Parkinson's disease-related pathology. Cell Tissue Res 2004; 318 (1): 121-34

11. Kish SJ, Shannak K, Hornykiewicz O. Uneven pattern of dopamine loss in the striatum of patients with idiopathic Parkinson's disease. Pathophysio-logic and clinical implications. N Engl J Med 1988; 318 (14): 876-80

12. Albin RL, Young AB, Penney JB. The functional anatomy of basal ganglia disorders. Trends Neu-rosci 1989; 12 (10): 366-75.

13. Albin RL, Young AB, Penney JB. The functional anatomy of disorders of the basal ganglia. Trends Neurosci 1995; 18 (2): 63-4.

14. Naito $A$, Kita $H$. The cortico-nigral projection in the rat: an anterograde tracing study with biotin-ylated dextran amine. Brain Res 1994 Feb 21; 637 (1-2): 317-22.

15. Fogelson N, Pogosyan A, Kuhn AA, Kupsch A, van $B G$, Speelman $H$, et al. Reciprocal interactions between oscillatory activities of different frequencies in the subthalamic region of patients with Parkinson's disease. Eur J Neurosci 2005; 22 (1): 257-66.

16. Pan MK, Tai CH, Kuo CC. Parkinson's disease and cortico-Basal Ganglia circuits. Acta Neurol Taiwan 2010; 19 (3): 209-18.

17. Smith Y, Parent A. Neurons of the subthalamic nucleus in primates display glutamate but not GABA immunoreactivity. Brain Res 1988; 453 (12): 353-6.

18. Albin RL, Greenamyre JT. Alternative excitotoxic hypotheses. Neurology 1992; 42 (4): 733-8.

19. Rodriguez MC, Obeso JA, Olanow CW. Subthalamic nucleus-mediated excitotoxicity in Parkinson's disease: a target for neuroprotection. Ann Neurol 1998; 44 (3 Suppl 1): S175-S188.

20. Hughes AJ, Daniel SE, Kilford L, Lees AJ. Accuracy of clinical diagnosis of idiopathic Parkinson's disease. A clinico-pathological study of 100 cases.

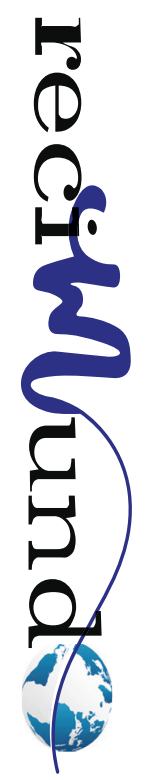




\section{VALLEJO ZAMBRANO, C. R., JIMÉNEZ JIMÉNEZ, R. A., MORÁN RODRÍGUEZ, V. E., GÓMEZ CHUMO, M. E., DEL VALLE PILAY, M. B., \& PALMA MORENO, N. J.}

J Neurol Neurosurg Psychiatry 1992;55:181-4.

21. Chaudhuri KR, Schapira AH. Non-motor symptoms of Parkinson's's disease: Dopaminergic pathophysiology and treatment. Lancet Neurol 2009;8:464-74.

22. Jankovic J, McDermott M, Carter J, Gauthier S, Goetz C, Golbe L, et al. Variable expression of Parkinson's disease: A base-line analysis of the DATATOP cohort. The Parkinson's Study Group. Neurology 1990;40, 1529-34.

23. Lim SY, Lang AE. The nonmotor symptoms of Parkinson's disease-An overview. Mov Disord 2010;25(Suppl 1): S123-S130.

24. Aarsland D, Beyer MK, Kurz MW. Dementia in Parkinson's disease. Curr Op Neurol 2008;21:67682.

25. Postuma RB, Berg D, Stern M, Poewe W, Olanow CW, Oertel W, et al. MDS clinical diagnostic criteria for Parkinson's disease. Movement disorders: official journal of the Movement Disorder Society. 2015;30(12):1591-601.

26. Rizzo G, Copetti M, Arcuti S, Martino D, Fontana A, Logroscino G. Accuracy of clinical diagnosis of Parkinson's disease: A systematic review and meta- analysis. Neurology 2016 86:566-76.

27. Politis M. Neuroimaging in Parkinson's disease: From research setting to clinical practice. Nat Rev Neurol 2014;10:708-22

28. Ibrahim N, Kusmirek J, Struck AF, Floberg JM, Perlman SB, Gallagher C, et al. The sensitivity and specificity of F-DOPA PET in a movement disorder clinic. Am J Nucl Med Mol Imaging 2016;6:102-9.

29. Chen-Plotkin AS. Unbiased approaches to biomarker discovery in neurodegenerative diseases. Neuron 2014;84:594-607.

30. Ferreira JJ, Katzenschlager R, Bloem BR, Bonuccelli $U$, Burn D, Deuschl $G$, et al. Summary of the recommendations of the EFNS/MDS-ES review on therapeutic management of Parkinson's disease. European journal of neurology. 2013;20(1): 5 -15.

31. Meissner WG, Frasier M, Gasser T, Goetz CG, Lozano A, Piccini P, et al. Priorities in Parkinson's disease research. Nature reviews Drug discovery. 2011;10(5):377-93

32. Miyasaki JM, Martin W, Suchowersky O, Weiner WJ, Lang AE. Practice parameter: Initiation of treatment for Parkinson's disease: An evidence-based review: Report of the quality standards subcommittee of the American Academy of Neurology. Neu- rology 2002;58:11-17

33. Poewe W., Antonini A. Novel formulations and modes of delivery of levodopa. Mov Disord 2015;30:114-20.

34. Fox SH, Katzenschlager R, Lim S-Y, Ravina B, Seppi K, Coelho M, et al. The Movement Disorder Society evidence-based medicine review update: Treatments for the motor symptoms of Parkinson's disease. Mov Disord 2011;26:S2-41.

35. Connolly B, Lang AE. Pharmacological treatment of Parkinson's disease: A review. JAMA 2014;311:1670-83.

36. Kalia LV, Brotchie JM, Fox SH. Novel nondopaminergic targets for motor features of Parkinson's's disease: Review of recent trials. Mov Disord 2013;28:131-44

37. Seppi, K, Weintraub D, Coelho M, Perez-Lloret S, Fox SH, Katzenschlager R, et al. The Movement Disorder Society evidence-based medicine review update: Treatments for the non-motor symptoms of Parkinson's disease. Mov Disord 2011;26:S42-S80.

38. Connolly B, Fox SH. Treatment of cognitive, psychiatric, and affective disorders associated with Parkinson's disease. Neurotherapeutics 2014;11:78-91.

39. Zhou, ZH, Wu YF, Wang X, Hahn YZ. The c-Abl inhibitor in Parkinson's disease. Neurol Sci 2017;38:547-52.

40. Martinez-Fernandez, R., Gasca Salas, C., Sanchez Ferro, A., Obeso, J., 2016. Actualizaciónen la enfermedad de parkinson. Rev. Med. Clin. Condes 27 (3), 363-379.

41. Lozano CS, Tam J, Lozano AM (2018) The changing landscape of surgery for Parkinson's disease. Mov Disord 33, 36-47.

42. Hassler R, Riechert T (1954) [Indications and localization of stereotactic brain operations]. Nervenarzt 25, 441-447.

43. Follett KA, Weaver FM, Stern M, Hur K, Harris CL, Luo P, Marks WJ, Jr., Rothlind J, Sagher O, Moy C, Pahwa R, Burchiel K, Hogarth P, Lai EC, Duda JE, Holloway K, Samii A, Horn S, Bronstein JM, Stoner G, Starr PA, Simpson R, Baltuch G, De Salles A, Huang GD, Reda DJ, CSP 468 Study Group (2010) Pallidal versus subthalamic deepbrain stimulation for Parkinson's disease.N Engl J Med 362, 20772091.

44. Martinez-Fernandez R, Rodriguez-Rojas R, Del Alamo M, Hernandez-Fernandez F, Pineda-Pardo JA, Dileone M, Alonso-Frech F, Foffani G, Obeso 
I, Gasca-Salas C, de Luis-Pastor E, Vela L, Obeso JA (2018) Focused ultrasound subthalamotomy in patients with asymmetric Parkinson's disease: A pilot study. Lancet Neurol 17, 54-63.

45. Bond AE, Shah BB, Huss DS, Dallapiazza RF, Warren $A$, Harrison $M B$, Sperling $S A$, Wang $X Q$, Gwinn R, Witt J, Ro S, Elias WJ (2017) Safety and efficacy of focused ultrasound thalamotomy for patients with medication-refractory, tremor-dominant Parkinson disease: A randomized clinical trial. JAMA Neurol 74, 1412-1418.

46. Lee, Darrin J. and Lozano, Andres M. 'The Future of Surgical Treatments for Parkinson's Disease'. 1 Jan. 2018 : S79 - S83.

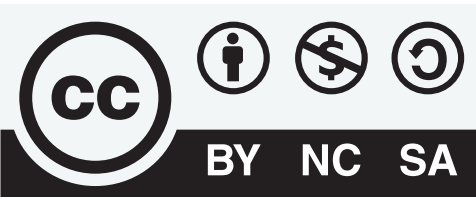

CREATIVE COMMONS RECONOCIMIENTO-NOCOMERCIAL-COMPARTIRIGUAL 4.0.

\section{CITAR ESTE ARTICULO:}

Vallejo Zambrano, C. R., Jiménez Jiménez, R. A., Morán Rodríguez, V. E., Gómez Chumo, M. E., Del Valle Pilay, M. B., \& Palma Moreno, N. J. (2020). Síndrome de Parkinson: Revisión bibliográfica y actualización. RECIMUNDO, 4(4), $270-$ 281. https://doi.org/10.26820/recimundo/4.(4).octubre.2020.270-281 